\title{
Anatomical and Functional Outcome of Epiretinal Membrane Peeling Surgery
}

URMISH V GOSWAMI ( $D$ urmishgoswami@gmail.com )

BJ Medical College https://orcid.org/0000-0002-5586-0556

Gosai Jignesh Y

Government Eye Hospital, Civil Hospital

Modi Pratik A

Government Eye Hospital, Civil Hospital

\section{Research Article}

Keywords: Retina, Macula, Best corrected visual acuity(BCVA), Epiretinal membrane(ERM), Optical coherence tomography(OCT), Electroretinogram(ERG), Central Foveal Thickness(CFT)

Posted Date: September 27th, 2021

DOI: https://doi.org/10.21203/rs.3.rs-556364/v1

License: @ (i) This work is licensed under a Creative Commons Attribution 4.0 International License. Read Full License 


\section{Abstract}

Background: The purpose of this study is to study the anatomical and functional outcome of epiretinal membrane peeling surgery by evaluating the visual outcomes, comparing the pre and post operative macular thickness and functional changes through electroretinogram.

Methods: It is a non-randomized, open labelled, prospective study conducted in a tertiary eye care centre. The anatomical and functional outcome of epiretinal membrane peeling surgery was evaluated by comparing the preoperative BCVA, OCT and ERG with post operative results of the same, at $4 \& 12$ weeks after the surgery in 25 eyes.

Results: There was a significant reduction in CFT $(p \leq 0.05)$ with marked improvement in vision on follow ups at 4 weeks and 12 weeks after removal of ERM with significant reduction in the macular edema. On post operative ERG, 18 (72\%) patients showed global improvement in amplitudes and P1 implicit time as compared to their preoperative value while $2(8 \%)$ patients showed global improvement in amplitude but there was still delay in P1 implicit time as similar to their preoperative value and 4 patients (16\%) showed no improvement in global amplitude as well as in P1 implicit time.

Conclusions: ERM peeling surgery in selected cases improves visual acuity significantly in majority of the patients. CFT and foveal architecture significantly improves with ERM peeling surgery which also explains the improvement in functional visual acuity. ERG interpretation goes along the improvement in best corrected visual acuity signifying improvement in functioning of macula.

\section{Introduction}

An epiretinal membrane (ERM) is a thin sheet of fibrous tissue that can develop on the surface of the macular area of the retina and cause disturbance in vision. An epiretinal membrane can also be called macular pucker, premacular fibrosis, surface wrinkling retinopathy or cellophane maculopathy. Most commonly, macular epiretinal membrane are asymptomatic or causes mild symptoms of metamorphopsia and/or modest decrease in central visual acuity. A minority of these membranes can cause macular distortion or macular edema to induce clinicians to recommend ERM removal via pars plana vitrectomy.

Optical coherence tomography (OCT) has become the most useful, noninvasive ancillary test for the evaluation of epiretinal membranes. OCT may be predictive of visual outcomes with ERM surgery. The hyperreflective line above retinal pigment epithelium (RPE) demonstrates the inner segment/outer segment (IS/OS) junction of photoreceptors. Several studies have demonstrated that an intact continuous IS/OS junction is predictive of better visual outcome at 12 months. ${ }^{(1,2)}$ OCT also helps in distinguishing macular pseudo holes from full thickness macular hole which is an exclusion criterion for this study. ${ }^{(1)}$ The detection of elevated ERM via enface OCT could assist safer grasping of the ERM and indicate the potential for visual outcome improvement after PPV and ERM peeling. ${ }^{(3)}$ 
The multifocal electroretinogram ( $\mathrm{mfERG}$ ) represents a cone generated response of localized retinal function in the central macula, which is useful in establishing the presence of macular dysfunction. (4) Multifocal electroretinography (mfERG), is a noninvasive, objective method to detect regional functional changes in the central retina by measuring electrophysiologic responses, has demonstrated macular function changes in eyes with ERM by several reports. $(5,6)$ The biphasic first order response of the mfERG includes an initial negative deflection [N1] followed by a positive peak [P1]. Previous studies have found that abnormalities in the P1 latency disorders might reflect dysfunctions of the inner retinal layers and Müller cells. ${ }^{(7)}$

The mfERG values might be associated with numerous factors. In another study it was demonstrated that there was a significant correlation between P1 implicit time and CMT. MfERG abnormalities appear to demonstrate subtle macular function changes and correlate with visual acuity and central macular thickness in eyes with ERM. In first-order mfERG responses, P1 wave changes may be a sensitive functional measurement for ERM patients.

The goal of membrane peeling is to reduce or eliminate the most common mechanisms of visual loss, including macular distortion, traction macular detachment, foveal ectopia, tissue that covers the fovea, retinal vascular leakage with macular edema, and traction induced obstruction of axoplasmic flow. In general, most patients considered for vitrectomy have significant visual impairment.

ERMs are found most frequently over the age of 50 and several large clinical studies have noted a clinical prevalence lying between $7 \%$ and $11.8 \% .\left({ }^{(8,9)}\right.$ Most of these are asymptomatic, with many being extra foveal in location. There appears to be no significant gender predilection and 20 to $30 \%$ are bilateral. Other eye involvement was reported in the Blue Mountains Eye Study to occur in $13.5 \%$ of patients over a 5 years time period. ${ }^{(10)}$ The majority of ERMs are globally adherent to the retinal surface, however, some appear to have focal adhesions. ${ }^{(11)}$ These focal adhesions are more common in eyes with secondary ERMs.

The incidence of symptomatic ERM formation is $4-8 \%$ after repair of rhegmatogenous retinal detachment, ${ }^{(12,13)}$ and $1-2 \%$ after prophylactic treatment of peripheral retinal breaks. ${ }^{(14)}$

The electroretinography (ERG) is a diagnostic test that measures the electrical activity generated by neural and non-neuronal cells in the retina in response to a light stimulus. The electrical response is a result of a retinal potential generated by light-induced changes in the flux of transretinal ions, primarily sodium and potassium. Most often, ERGs are obtained using electrodes embedded in a corneal contact lens, which measure a summation of retinal electrical activity at the corneal surface. The International Society for Clinical Electrophysiology of Vision (ISCEV) introduced minimum standards for the ERG in 1989. The ERG can provide important diagnostic information on a variety of retinal disorders. Moreover, an ERG can also be used to monitor disease progression.

\section{Materials And Methods}


This is a non randomized, open labelled, prospective study conducted in a tertiary eye care centre. In this study 25 eyes are included as per the inclusion and exclusion criteria mentioned below.

In this study a detailed history and clinical examination were performed with necessary investigations as and when required. Pre-operatively patients were evaluated for Best Corrected Visual Acuity (BCVA), metamorphopsia, fundus examination with indirect ophthalmoscopy and slit lamp biomicroscopy, fundus photographs were taken of all patients. Optical Coherence Tomography (OCT) was performed preoperatively to assess the macular anatomy and to measure Central Foveal Thickness (CFT). Multifocal Electroretinogram (mfERG) was performed in all patients for preoperative evaluation of electrophysioloigc response of central retina. Patients in this study then underwent pars plana vitrectomy with epiretinal membrane peeling. BCVA was assessed at post-operative day 1 and post operative 1 week, 4 weeks and 12 weeks with focus also on improvement in the quality of the vision and reduction in metamorphopsia. OCT was performed at 4 weeks and 12 weeks to evaluate the change in macular anatomy and central foveal thickness after epiretinal membrane peeling surgery. ERG was performed in all patients 12 weeks after surgery to evaluate the electrophysiologic response of central retina post surgery. Thus in this study anatomical and functional outcome of epiretinal membrane peeling surgery was evaluated by comparing the preoperative BCVA, OCT and ERG with post operative results of the same, 12 weeks after the surgery.

The Ethical approval for the study was taken from the local Institutional Ethical Committee.

None of the authors have any financial/conflicting interests to disclose.

\section{Inclusion criteria:}

- Patients between the ages of 20 to 80 years.

- Intraocular pressure $<21 \mathrm{mmHg}$.

- Fundoscopy showing physiological cupping.

- Slit Lamp Examination showing: clear cornea, normal anterior chamber depth.

- Normal pupillary shape, size and reaction.

- Preoperative OCT presence of ERM on macular area covering central fovea with or without macular edema.

- Clearance of media for preoperative mfERG and OCT.

- Visual acuity - BCVA (Best Corrected Visual Acuity) </=6/12.

\section{Exclusion criteria:}

- Patient's age $<20$ years or $>80$ years.

- Higher Intraocular pressure $>21 \mathrm{mmHg}$.

- Poor fixation for OCT examination.

- Cataract, which precludes OCT. 
- Patients with pre-existing macular hole, optic atrophy, vitreomacular traction, choroidal neovascular membrane, macular ischemia from previous retinal vascular occlusion or any other pre existing macular disorder.

- Patients with rubeosis iridis or glaucoma.

- Only eyed patient.

- Uncontrolled diabetes.

\section{History}

Detailed clinical history of the patients were taken according to the proforma as mentioned below.

Clinical examination:

1. Vision with or without glasses

2. Torch light \& Slit lamp examination

3. IOP by Applanation tonometer

4. Fundoscopy by Slit lamp biomicroscopy using 90 D, Direct \& and Indirect Ophthalmoscopy

5. Optical coherence tomography (OCT)

6. Electroretinogram

Based on this, patients having epiretinal membrane were diagnosed and operated. Patients were followed up at day-1, 1 week, 4 weeks and 12 weeks and were assessed for best corrected visual acuity, evaluation of metamorphopsia, fundus examination, OCT and mfERG (pre-operative and post-operative at 12 weeks).

\section{Data Analysis (Results)}

A Non randomized, open labelled prospective study of 25 eyes of patients with epiretinal membrane of idiopathic origin as well as other causes were carried out at $\mathrm{M}$ and $\mathrm{J}$ Institute of Ophthalmology, Civil Hospital, Ahmedabad.

\section{DEMOGRAPHIC \& BASELINE RESULTS 1. AGE}

Of 25 patients, 1 patient was of 40 years of age, 2 were in 40-50 age group, 7 in 50-60 age group, 14 were in 60-70 age group, and 1 was above 70years of age. 


\begin{tabular}{|c|c|c|c|}
\hline AGEGROUP & Frequency & Percent & Cum. Percent \\
\hline$>30-40$ & 1 & $4.00 \%$ & $4.00 \%$ \\
\hline$>40-50$ & 2 & $8.00 \%$ & $12.00 \%$ \\
\hline$>50-60$ & 7 & $28.00 \%$ & $40.00 \%$ \\
\hline$>60-70$ & 14 & $56.00 \%$ & $96.00 \%$ \\
\hline$>70-80$ & 1 & $4.00 \%$ & $100.00 \%$ \\
\hline Total & 25 & $100.00 \%$ & $100.00 \%$ \\
\hline
\end{tabular}

\section{SEX}

In this study, 13 patients (52\%) were female \& 12 patients (48\%) were male.

\begin{tabular}{|lll|}
\hline SEX & Frequency & Percent \\
\hline F & 13 & $52.00 \%$ \\
\hline M & 12 & $48.00 \%$ \\
\hline Total & 25 & $100.00 \%$ \\
\hline
\end{tabular}

\section{BEST CORRECTED VISUAL ACUITY}




\begin{tabular}{|c|c|c|c|c|c|}
\hline SR.NO & PRE-OP & POD-1 & POST-OP 1 WEEK & POST-OP 4WEEKS & POST-OP 12 WEEKS \\
\hline 1 & 1.3 & 1.48 & 1.08 & 1 & 0.3 \\
\hline 2 & 0.48 & 1.78 & 0.6 & 0.18 & 0 \\
\hline 3 & 0.6 & 1.18 & 1 & 0.6 & 0.3 \\
\hline 4 & 0.3 & 1.08 & 0.6 & 0.3 & 0.3 \\
\hline 5 & 1.78 & 1.78 & 1 & 0.78 & 0.6 \\
\hline 6 & 0.3 & 1 & 0.78 & 0.48 & 0.18 \\
\hline 7 & 0.6 & 1.18 & 0.78 & 0.6 & 0.3 \\
\hline 8 & 1.48 & 1.78 & 1.3 & 1.48 & 1.08 \\
\hline 9 & 1.48 & 1.78 & 1.78 & 1.48 & 1.48 \\
\hline 10 & 1.78 & 1.78 & 1.3 & 1.08 & 1.08 \\
\hline 11 & 0.3 & 1 & 0.6 & 0.3 & 0.3 \\
\hline 12 & 2.48 & 1.78 & 1.78 & 1.78 & 1.78 \\
\hline 13 & 1.08 & 1.18 & 1 & 0.78 & 0.6 \\
\hline 14 & 1.18 & 2.48 & 1.18 & 1.08 & 2.48 \\
\hline 15 & 1.08 & 1.48 & 1.08 & 1 & 1 \\
\hline 16 & 1.08 & 1.18 & 0.78 & 0.78 & 0.48 \\
\hline 17 & 1.08 & 1.08 & 1 & 1 & 0.6 \\
\hline 18 & 1.48 & 1.3 & 1.18 & 1.18 & 1.18 \\
\hline 19 & 0.78 & 1.08 & 0.78 & 0.78 & 0.6 \\
\hline 20 & 1.18 & 1.18 & 1.08 & 1 & 1 \\
\hline 21 & 1 & 1.18 & 1 & 0.78 & 0.78 \\
\hline 22 & 0.78 & 1.08 & 1 & 0.6 & 0.6 \\
\hline 23 & 1.18 & 1.78 & 1.3 & 1.3 & 1.3 \\
\hline 24 & 0.78 & 1 & 0.78 & 0.78 & 0.6 \\
\hline 25 & 0.6 & 1 & 0.78 & 0.6 & 0.48 \\
\hline
\end{tabular}

In our study, post operative day 1 mean visual acuity improved from $1.04( \pm 0.52)$ to $1.38( \pm 0.38)$ which was statistically significant $(p=0.01)$, after 1 week mean VA was $1.02( \pm 0.31)$, which was statistically not 
significant $(p=0.87)$. After 4 weeks mean VA was $0.86( \pm 0.38)$, which was statistically significant $(p=$ $0.001)$. After 12 weeks mean VA was $0.72( \pm 0.45)$, which was statistically significant $(p=0.001)$.

\section{CENTRAL FOVEAL THICKNESS}




\begin{tabular}{|c|c|c|c|c|c|c|c|}
\hline $\begin{array}{l}\text { SR. } \\
\text { NO }\end{array}$ & $\begin{array}{l}\text { PRE- } \\
\text { OP }\end{array}$ & $\begin{array}{l}\text { POST- } \\
\text { OP } 4 \\
\text { WEEKS }\end{array}$ & $\begin{array}{l}\text { DIFFERENCE } \\
\text { (IN um) }\end{array}$ & $\begin{array}{l}\text { DIFFERENCE } \\
\text { (IN \%) }\end{array}$ & $\begin{array}{l}\text { POST-OP } \\
12 \\
\text { WEEKS }\end{array}$ & $\begin{array}{l}\text { DIFFERENCE } \\
\text { (IN um) }\end{array}$ & $\begin{array}{l}\text { DIFFERENCE } \\
\text { (IN \%) }\end{array}$ \\
\hline 1 & 245 & 234 & 11 & 4.49 & 225 & 20 & 8.16 \\
\hline 2 & 426 & 398 & 28 & 6.57 & 310 & 116 & 27.23 \\
\hline 3 & 383 & 345 & 38 & 9.2 & 248 & 135 & 35.24 \\
\hline 4 & 305 & 296 & 9 & 2.95 & 268 & 37 & 12.13 \\
\hline 5 & 591 & 433 & 158 & 26.73 & 290 & 301 & 50.93 \\
\hline 6 & 340 & 325 & 15 & 4.41 & 288 & 52 & 15.29 \\
\hline 7 & 375 & 345 & 30 & 8 & 300 & 75 & 20 \\
\hline 8 & 540 & 500 & 40 & 7.4 & 490 & 50 & 9.26 \\
\hline 9 & 308 & 290 & 18 & 5.84 & 282 & 26 & 8.44 \\
\hline 10 & 470 & 413 & 57 & 12.12 & 362 & 108 & 22.98 \\
\hline 11 & 324 & 288 & 36 & 11.11 & 240 & 84 & 25.92 \\
\hline 12 & 320 & 300 & 20 & 6.25 & 300 & 20 & 6.25 \\
\hline 13 & 459 & 408 & 51 & 11.11 & 300 & 159 & 33.98 \\
\hline 14 & 289 & 275 & 14 & 4.84 & 370 & NA & ERR \\
\hline 15 & 386 & 350 & 36 & 9.33 & 298 & 88 & 22.79 \\
\hline 16 & 373 & 354 & 19 & 5.09 & 302 & 71 & 19.03 \\
\hline 17 & 478 & 423 & 55 & 11.51 & 288 & 190 & 39.74 \\
\hline 18 & 465 & 438 & 27 & 5.8 & 424 & 41 & 8.82 \\
\hline 19 & 389 & 342 & 47 & 12.08 & 298 & 91 & 23.39 \\
\hline 20 & 460 & 430 & 30 & 6.52 & 356 & 104 & 22.61 \\
\hline 21 & 389 & 355 & 34 & 8.74 & 296 & 93 & 23.9 \\
\hline 22 & 280 & 268 & 12 & 4.28 & 250 & 30 & 10.71 \\
\hline 23 & 436 & 414 & 22 & 5.05 & 398 & 38 & 8.71 \\
\hline 24 & 378 & 348 & 30 & 7.93 & 286 & 92 & 24.39 \\
\hline 25 & 295 & 268 & 27 & 9.15 & 243 & 52 & 17.62 \\
\hline
\end{tabular}


In our study above table shows data of 25 patients who were operated \& their Central foveal thickness changes in follow ups at 4 weeks and 12 weeks. Overall, there was a significant improvement in CFT statistically $(p=<0.05)$.

In our study after 4 weeks mean CFT improved from $388 \mathrm{~mm}( \pm 88.16)$ to $353 \mathrm{~mm}( \pm 67.21)$ \& $8.26 \%$ (35 $\mathrm{mm}$ ) improvement which was statistically significant also $(\mathrm{p}=0.0006)$, after 12 weeks mean CFT was $308.48 \mathrm{~mm}( \pm 61.54)$ \& improvement $20.73 \%(79.52 \mathrm{~mm})$ which was statistically significant $(p=0.0006)$.

\section{RELATION BETWEEN CFT AND BCVA IN LOG MAR}

The below scatter graph shows association between central foveal thickness pre-operative and after removal of ERM at 4 weeks and 12 weeks follow-up. It shows significant relation between BCVA and CFT and also shows marked improvement in vision with reduction in CFT after removal of ERM as the traction at the fovea is released as well as significant reduction in the macular edema.

\section{ERG INTERPRETATION}

In our study 18 (72\%) patients showed global improvement in amplitudes and P1 implicit time as compared to their preoperative value while $2(8 \%)$ patients showed global improvement in amplitude but there was still delay in P1 implicit time as similar to their preoperative value and 4 patients (16\%) showed no improvement in global amplitude as well as in P1 implicit time.

\section{SURGICAL COMPLICATIONS}

\begin{tabular}{|ll|}
\hline Complications & Number of patients \\
\hline Vitreous Haemorrhage & 2 \\
\hline Macular Edema & 4 \\
\hline Raised IOP & 5 \\
\hline Endophthalmitis & 1 \\
\hline Macular Haemorrhages & 3 \\
\hline None & 10 \\
\hline
\end{tabular}

Out of the 25 patients taken for the study, 2 patients (8\%) had vitreous haemorrhage on post operative day 1 which resolved spontaneously with conservative management. 4 patients (16\%) had persistent macular edema, 5 patients $(20 \%)$ had raised intraocular pressure which was managed by topical anti glaucoma drugs. 3 patients (12\%) showed haemorrhages at macular area after epiretinal membrane peeling, 10 patients $(40 \%)$ had uneventful surgery. One of the patients developed post operative endopthalmitis at 12 weeks which was managed with intravitreal as well as systemic antibiotics. This patient later showed signs of pre-phthysical eye as suggested by ultrasonography. 


\section{Discussion}

In the study conducted by James G Wong MMed, Nitin Sachdev PhD, Paul E Beaumont FRANZCO, Andrew A Chang FRANZCO of "Visual outcomes following vitrectomy and peeling of epiretinal membrane" in 125 eyes of 123 patients with visually significant macular epiretinal membranes showed Visual acuity improved by a mean of $0.31 \hat{A} \pm 0.21$ logMAR (three lines of vision). ${ }^{(15)}$ Whereas our study which showed improvement by mean of $0.72( \pm 0.45) \log M A R$. As mentioned in the study, we observed that epiretinal membrane peeling improves vision in the majority of patients with significant symptoms, even if preoperative visual acuity is not substantially reduced. Surgery improves functional vision including metamorphopsia not measurable by visual acuity, and thus assessment of functional vision should be included in surgical case planning.

In a similar study conducted by lonas Miliatos 1 and Gun Lindgren2 of Epiretinal membrane surgery evaluated by subjective outcome in 239 eyes of 231 patients with idiopathic ERM showed that the preoperative visual acuity was mean $0.58 \log M A R$ which improved to post operative visual acuity of mean $0.36 \log M A R$ which was very significant with $p$ value $<0.05$. ${ }^{(16)}$ Where as our study showed improvement in visual acuity from mean $1.04 \log$ MAR pre-operratively to visual acuity of mean 0.72 $\log M A R$ which is also significant with $p$ value of 0.001 .

In a study conducted by Parisi V, Coppe AM, Gallinaro G, Strip M of "Assessment of macular function by focal electroretinogram and pattern electroretinogram before and after epimacular membrane surgery assessment of macular volume" were evaluated for 22 eyes of 22 patients (mean age +/- SD, 63.20 +/10.0 years) with ERM preoperatively (baseline) and 6 months after surgical peeling. Preoperative visual acuity and fERG and pERG amplitudes observed in ERM eyes were compared with those in 15 agematched control eyes. It was observed that, at the postoperative evaluation, ERM eyes had a correlated significant $(P<0.01)$ increase in visual acuity, fERG amplitude, and $p E R G$ amplitude with respect to the preoperative values. All ERM eyes had a significant $(\mathrm{P}<0.01)$ reduction in macular volume, and retinal microanatomy was restored to normal conditions. Hence the decrease in visual acuity is related to dysfunction of both preganglionic (abnormal fERG) and ganglionic (abnormal pERG) macular elements. Surgical removal of ERM may induce improvement of the function of both outer and innermost macular retinal layers, leading to a related increase in visual acuity. ${ }^{(17)}$ In our study we performed preoperative and post operative multifocal ERGs in 25 eyes of 25 patients which showed improvement in global and $P$ wave amplitudes as well improvement in delay in P1 implicit time post ERM peeling surgery correlated with improvement in visual acuity.

In a study conducted by Liu Z, Ye J, Chen Y, Dong F of "Clinical study of vitrectomy with epiretinal membrane peeling for idiopathic macular membrane" Clinical data of 51 patients (51 eyes) who underwent vitrectomy with ERM peeling were retrospectively investigated. All the patients were examined by visual acuity, slit lamp, fundus under mydriasis, optical coherence tomography (OCT) before and after the surgery. The 3-18 months follow-up were included. There was a negative correlation between visual acuity and mean foveomacular thickness $(r=-0.452, P=0.001)$, and it was obvious postoperatively 
$(r=-0.602, P=0.000)$. The increase of visual acuity was strongly correlated with the decline of mean foveomacular thickness $(r=0.382, P=0.006)$. It was concluded that Vitrectomy with ERM peeling can improve visual acuity and ease macular edema. And it is a safe and effective therapy to treat patients. (18) In our study as mentioned in the scatter graph we observed the significant negative correlation between visual acuity and central foveal thickness both preoperatively and postoperatively at the end of 3 months.

In another study conducted by Soon II Kwon, Sung Jo Ko and In-Won Park of "The Clinical Course of the Idiopathic Epiretinal Membrane After Surgery" in 30 eyes of 30 patients and followed for a period of 7 months after surgery it was observed that there was significant improvement in the visual acuity with upto 2 or more lines of improvement and the mean foveal thickness which was 409.7+/-107.9 micron before surgery was improved to $288.6+/-66.1$ micron seven months after surgery. Thus the Foveal thickness and visual acuity improved until seven months after the vitrectomy in patients with idiopathic ERM. Preoperative visual acuity, foveal thickness, and final foveal thickness had a significant correlation with the final visual acuity. ${ }^{(19)}$ Whereas in our study the preoperative mean central foveal thickness $388 \mathrm{~mm}( \pm 88.16)$ was reduced to $308.48 \mathrm{~mm}( \pm 61.54)$ correlating with the improvement in visual acuity.

A study conducted by Jong-uk Hwang, Joonhong Sohn, Byung Gil Moon, Soo Geun Joe, Joo Yong Lee, June-Gone Kim, and Young Hee Yoon of "Assessment of Macular Function for Idiopathic Epiretinal Membranes Classified by Spectral-Domain Optical Coherence Tomography" showed that multifocal electroretinography can be used to investigate the pathophysiology of ERM and to evaluate the degree of functional demise in the fovea on SD-OCT. On the basis of Spectral Domain OCT they classified the patients in 2 groups depending on the anatomical distortion seen on SD-OCT. Among the two groups they observed preoperative reduction in N1 and P1 amplitudes which after surgical removal of epiretinal membrane showed improvement in amplitudes of ERGs significantly correlated with improvent in BCVA post surgery. ${ }^{(20)}$ Our study also shows improvement in amplitudes in mfERG consistent with improvement in BCVA with restoration of near normal anatomical configuration of macula.

\section{Conclusion}

1. ERM peeling surgery in selected cases improves visual acuity significantly in majority of the patients.

2. CFT and foveal architecture significantly improves with ERM peeling surgery which also explains the improvement in functional visual acuity .

3. ERG interpretation goes along the improvement in best corrected visual acuity signifying improvement in functioning of macula.

\section{References}

1. Iwanoff A: Beitrage zur normalen und pathologischen anatommie des auges.Grafes Arch ClinExp Ophthalmol 1865; 111135-170 
2. Roth AM, Foos RY: Surface wrinkling retinopathy in eyes enucleated atautopsy. Trans Am Acad Ophthalmol Otolaryngol 1971; 7511047-1059

3. Francois J, Verbraeken $\mathrm{H}$ : Relationship between the drainage of thesubretinal fluid in retinal detachment surgery and the appearance of macularpucker. Ophthalmologica 1979; 1791111-114

4. Tanenbaum HL, Schepens CL, Elzeneiny I, Freeman HM: Macular puckerfollowing retinal detachment surgery. Arch Ophthalmol 1970; 831286-293

5. Hwang JU, Sohn J, Moon BG, Joe SG, Lee JY, Kim JG, Yoon YH.Assessment of macular function for idiopathic epiretinal membranes classified by spectral-domain optical coherence tomography.Invest Ophthalmol Vis Sci. 2012 Jun 14;53(7):3562-9. doi: 10.1167/iovs.12-9762

6. Li D, Horiguchi M, Kishi S (2004) Tomographic and multifocal electroretinographic features of idiopathic epimacular membranes. Arch Ophthalmol 122(10):1462-1467.

doi:10.1001/archopht.122.10.1462

7. Lim JW, Kim HK, Cho DY (2011) Macular function and ultrastructure of the internal limiting membrane removed during surgery for idiopathic epiretinal membrane. Clin Experiment Ophthalmol 39(1):9-14

8. Mitchell P, Smith W, Chey T et al: Prevalence and associations of epiretinalmembranes. The Blue Mountains Eye Study, Australia. Ophthalmology 1997;1022-1040

9. Klein R, Klein BE, Wang Q et al (1994) The epidemiology of epiretinal membranes. Trans Am Ophthalmol Soc 92:403-425

10. Fraser-Bell S, Guzowski M, Rochtchina E et al: Five-year cumulativeincidence and progression of epiretinal membranes: the Blue Mountains EyeStudy.Ophthalmology 2003; 110134-40

11. Mori K, Gehlbach PL, Sano A et al (2004) Comparison of epiretinal membranes of differing pathogenesis using optical coherence tomography. Retina 24:57-62 A.

12. Hagler WS, Aturaliya U. Macular puckers after retinal detachment surgery. Br J Ophthalmol

13. Uemura A, Ideta H, Nagasaki H et al (1992) Macular pucker after retinal detachment surgery. Ophthalmic Surg 23:116-119 A.

14. Michels RG, Wilkinson CP, Rice TA (1990) Retinal detachment. CV Mosby, S. Louis, pp 1096-1098

15. Wong JG, Sachdev N, Beaumont PE, Chang AA (2005) Visual outcomes following vitrectomy and peeling of epiretinal membrane. Clinical Experimental ophthalmology 33:373-378. doi:10.1111/j.1442-9071.2005.01025.x

16. Miliatos I, Lindgren G. Epiretinal membrane surgery evaluated by subjective outcome.Acta Ophthalmol. 2017 Feb;95(1):52-59. doi: 10.1111/aos.13001. Epub 2016 Apr 4

17. Parisi V, Coppè AM, Gallinaro G, Stirpe M. Assessment of macular function by focal electroretinogram and pattern electroretinogram before and after epimacular membrane surgery.Retina. 2007 Mar;27(3):312-20

18. Liu Z, Ye J, Chen Y, Dong F [Clinical study of vitrectomy with epiretinal membrane peeling for idiopathic macular epiretinal membrane].Zhonghua Yan Ke Za Zhi (2016 May;52(5):343-7) doi: 
10.3760/cma.j.issn.0412-4081.2016.05.006. Chinese

19. Kwon SI, Ko SJ (2009 Dec) Park IW:The clinical course of the idiopathic epiretinal membrane after surgery. Korean J Ophthalmol 23(4):249-252. doi:10.3341/kjo.2009.23.4.249. Epub 2009 Dec 4

20. Hwang JU, Sohn J, Moon BG, Joe SG, Lee JY, Kim JG, Yoon YH.Assessment of macular function for idiopathic epiretinal membranes classified by spectral-domain optical coherence tomography.Invest Ophthalmol Vis Sci. 2012 Jun 14;53(7):3562-9. doi: 10.1167/iovs.12-9762

\section{Figures}

\section{Comparision of LogMAR Mean}

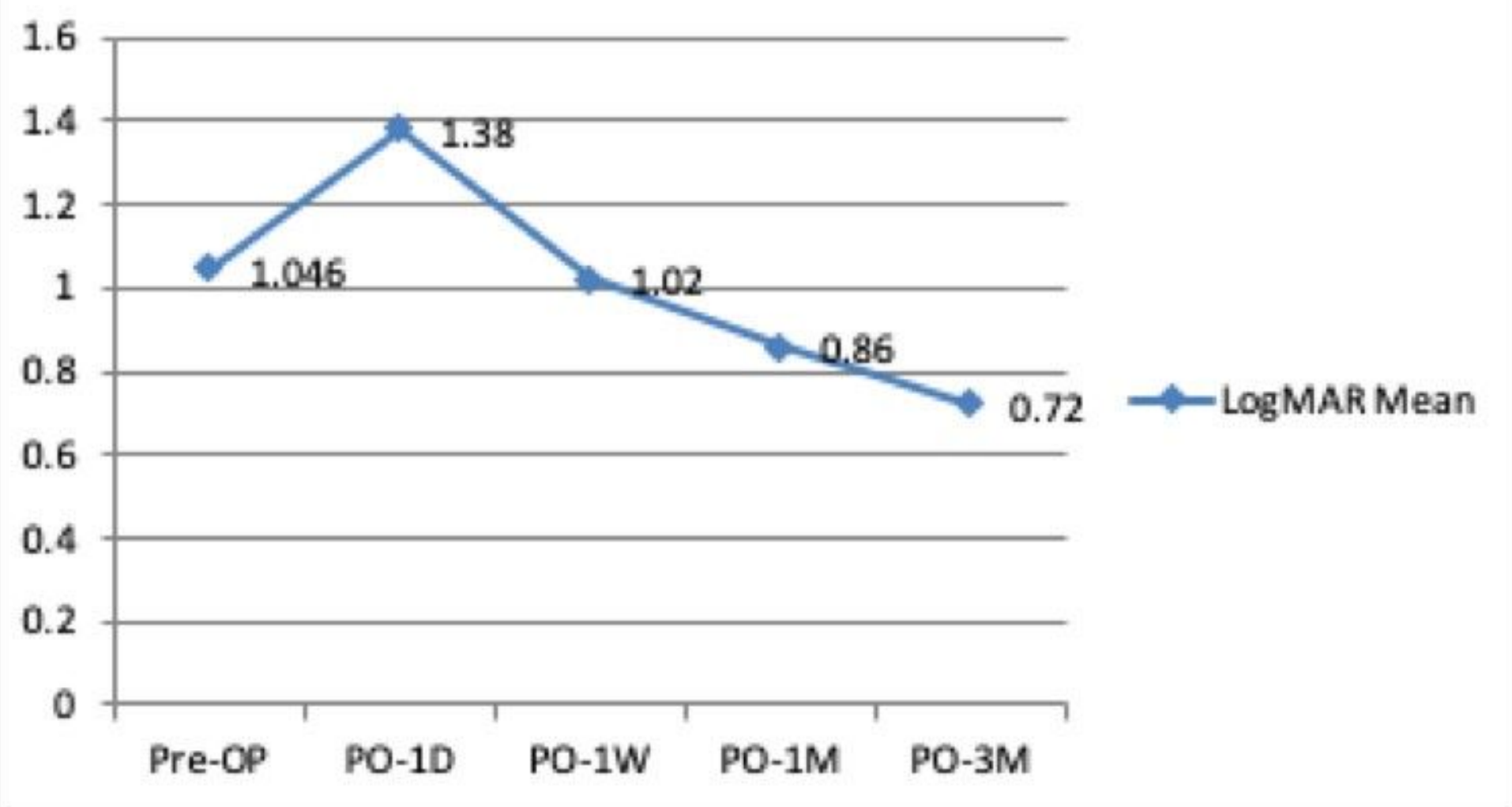

Figure 1

log mar mean visual acuity 


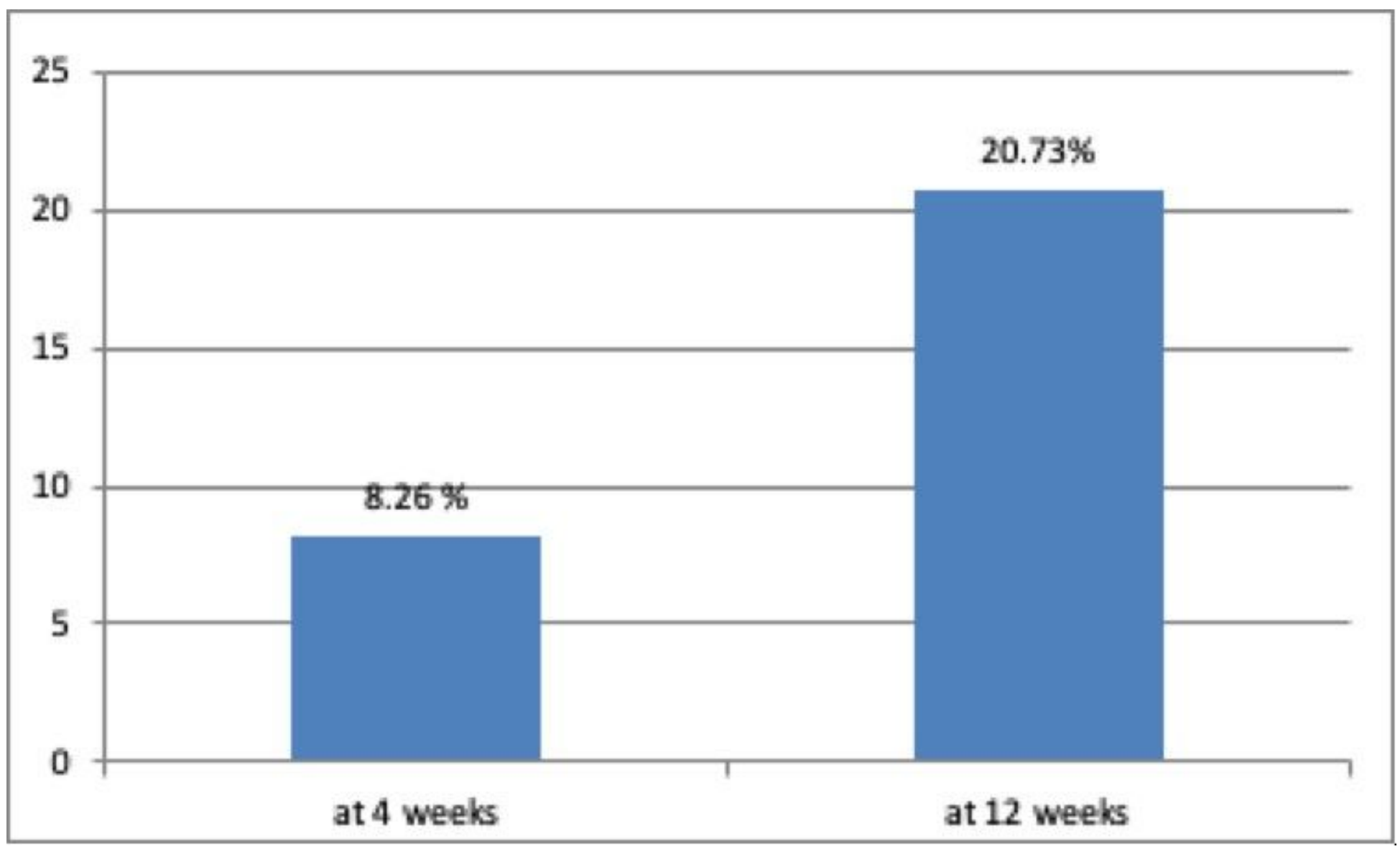

Figure 2

CFT at 412 wks 


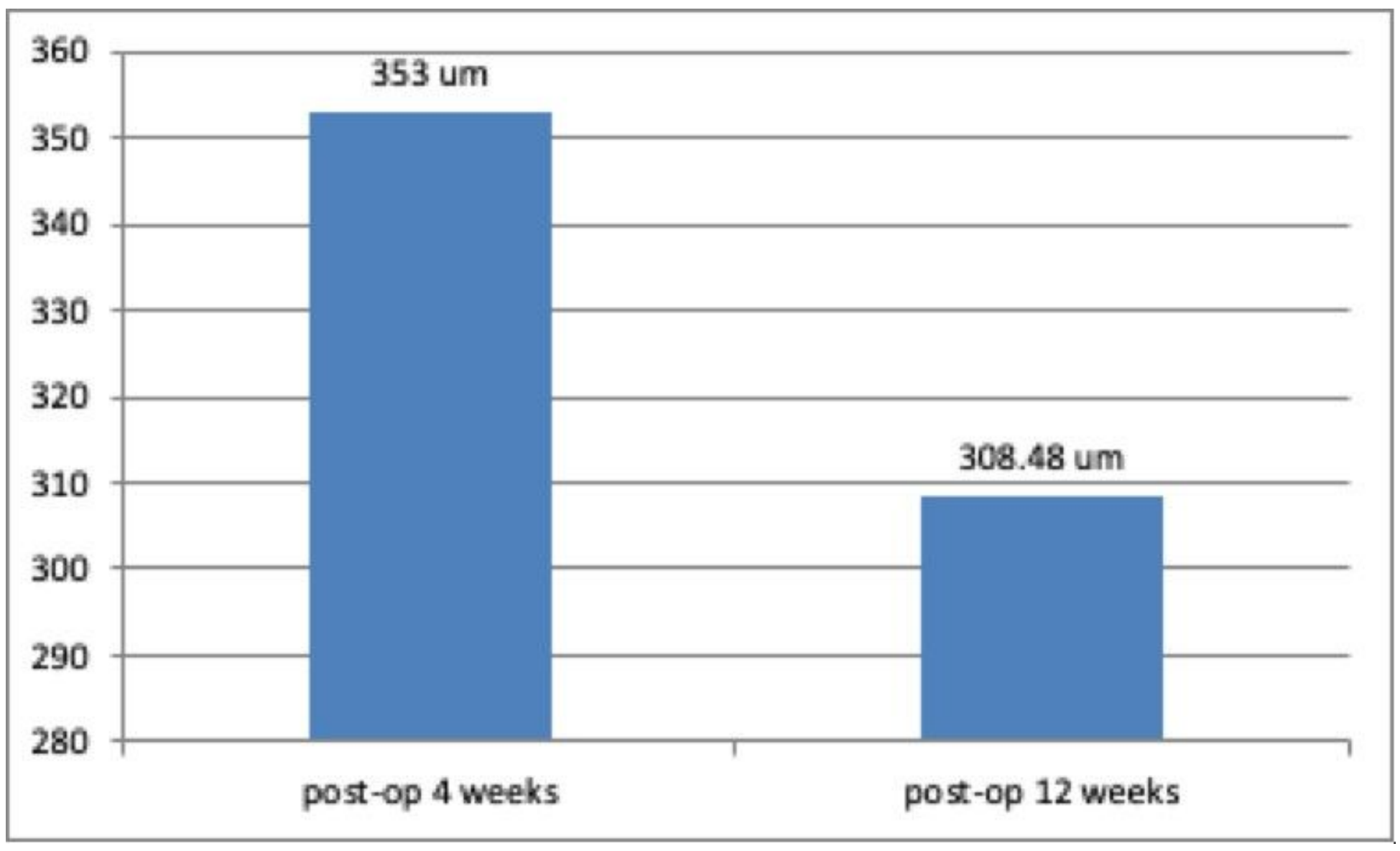

Figure 3

postop CFT

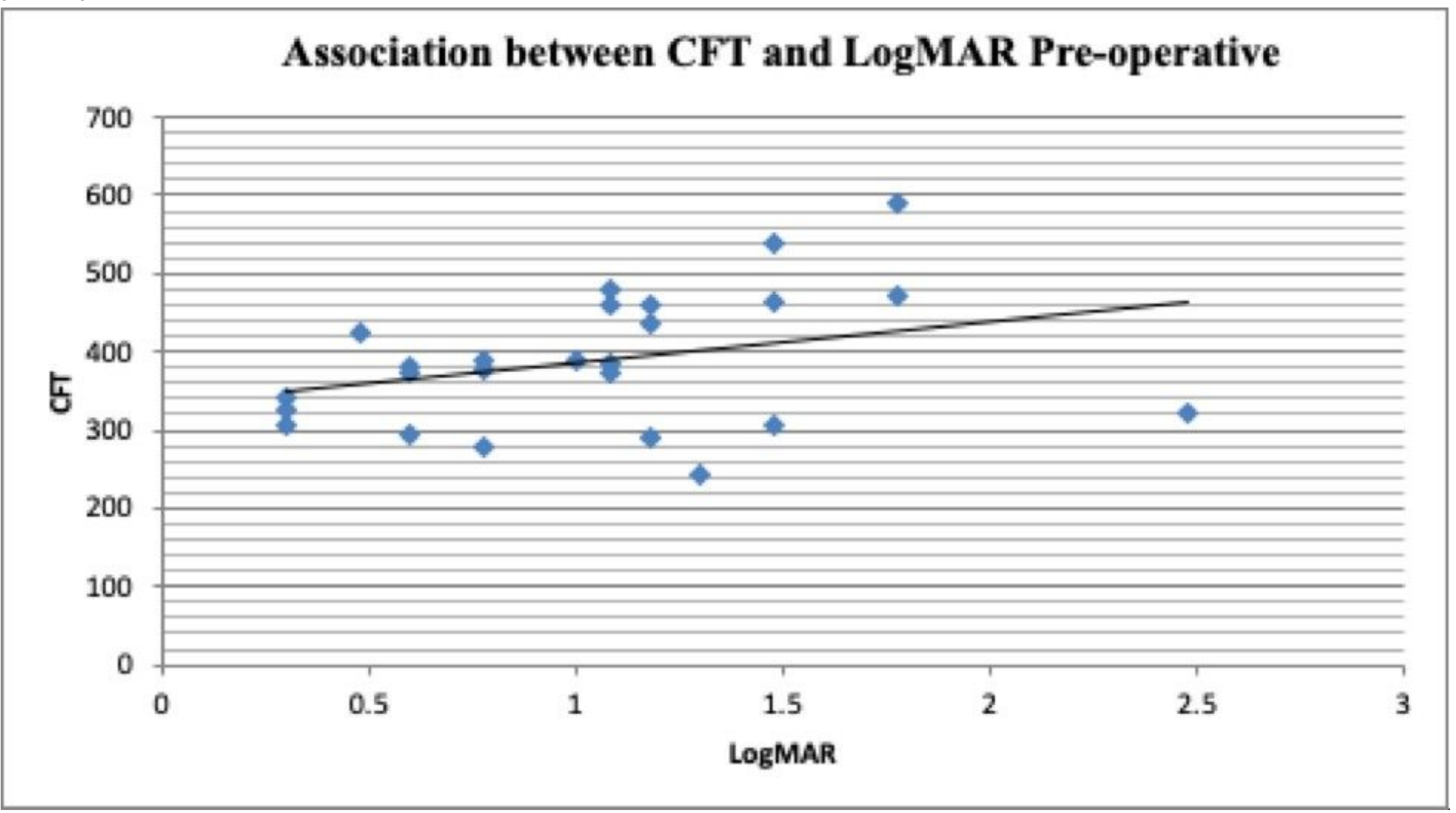


Figure 4

CFT preop

\section{Association between CFT and LogMAR Post-operative 3M}

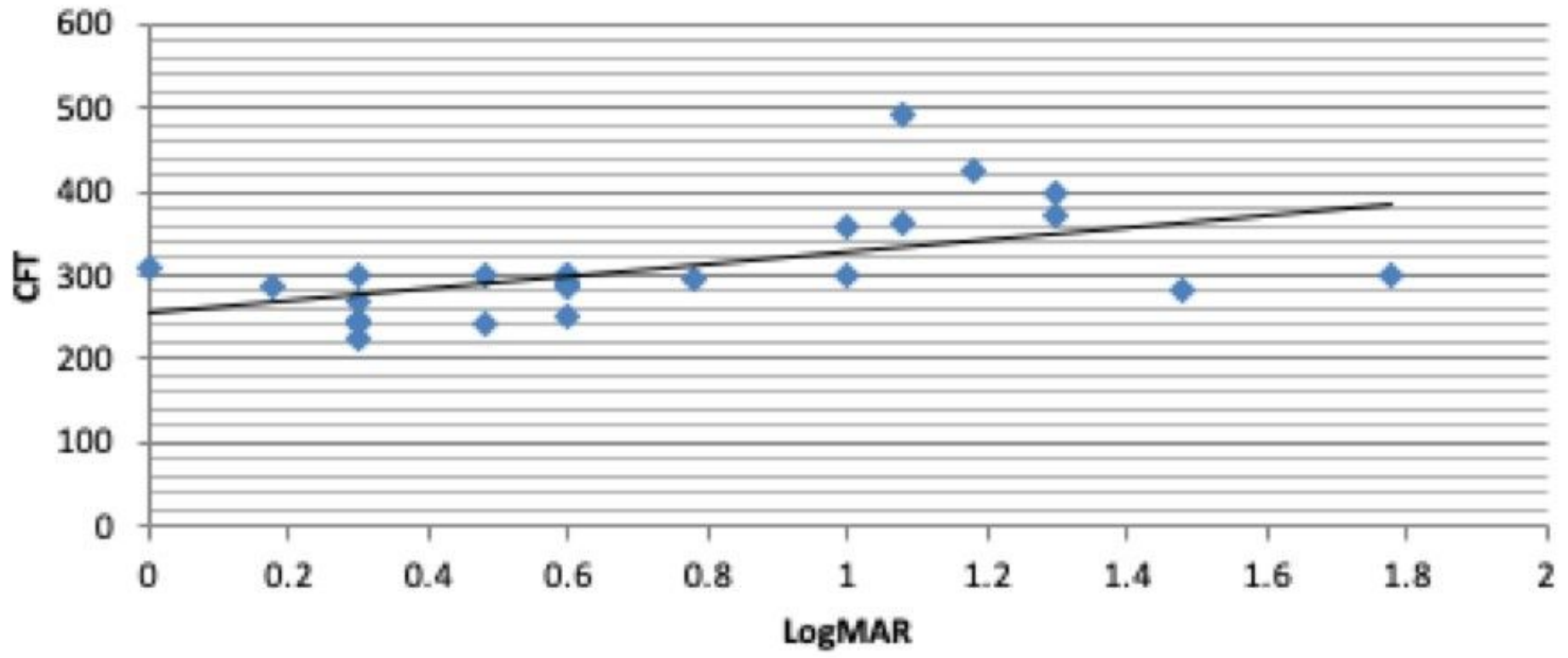

Figure 5

CFT 3mo postop

\section{Association between CFT and LogMAR Post-operative 1M}

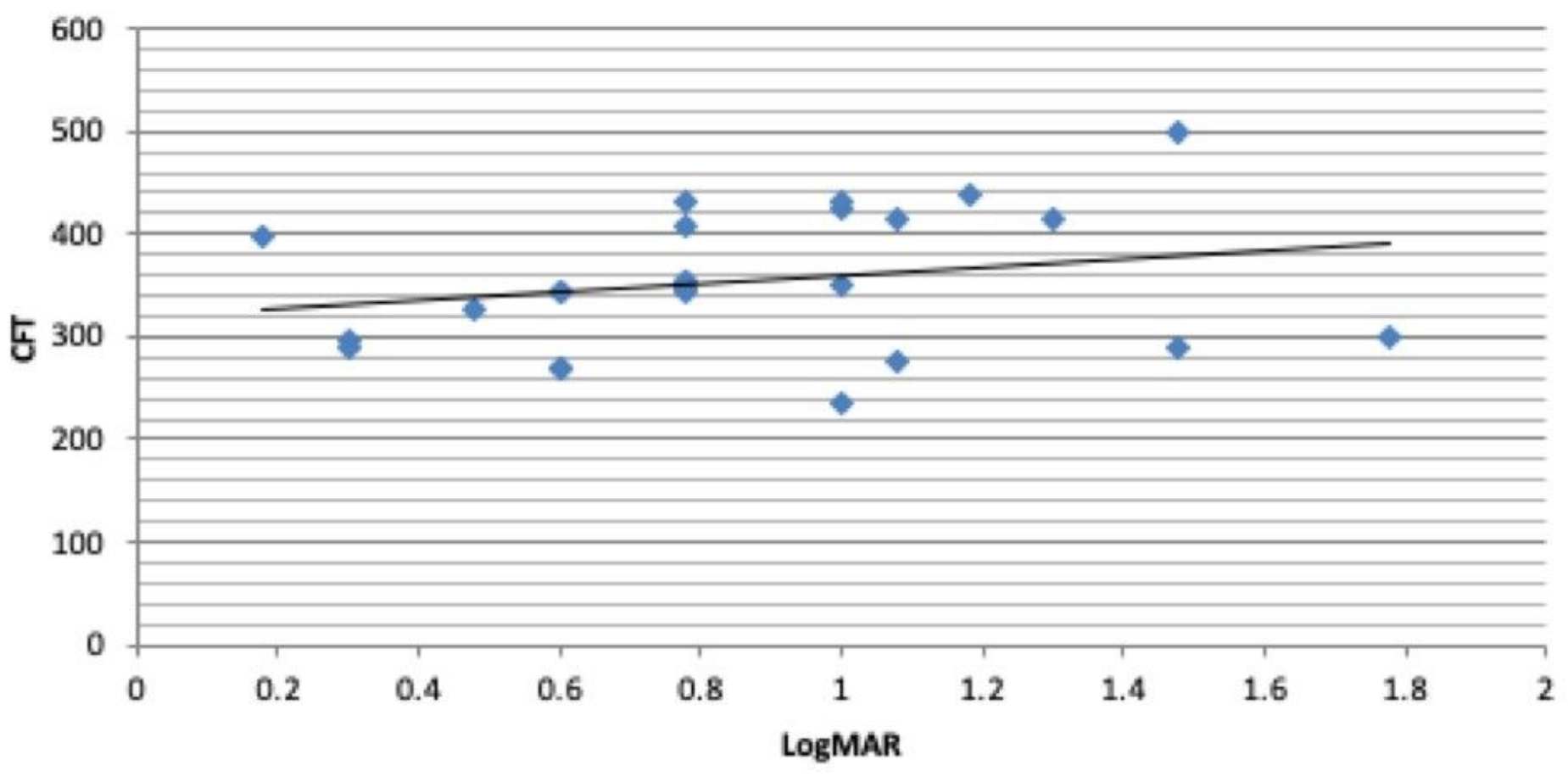


Figure 6

CFT postop 1 month

\section{Surgical \\ Complications}

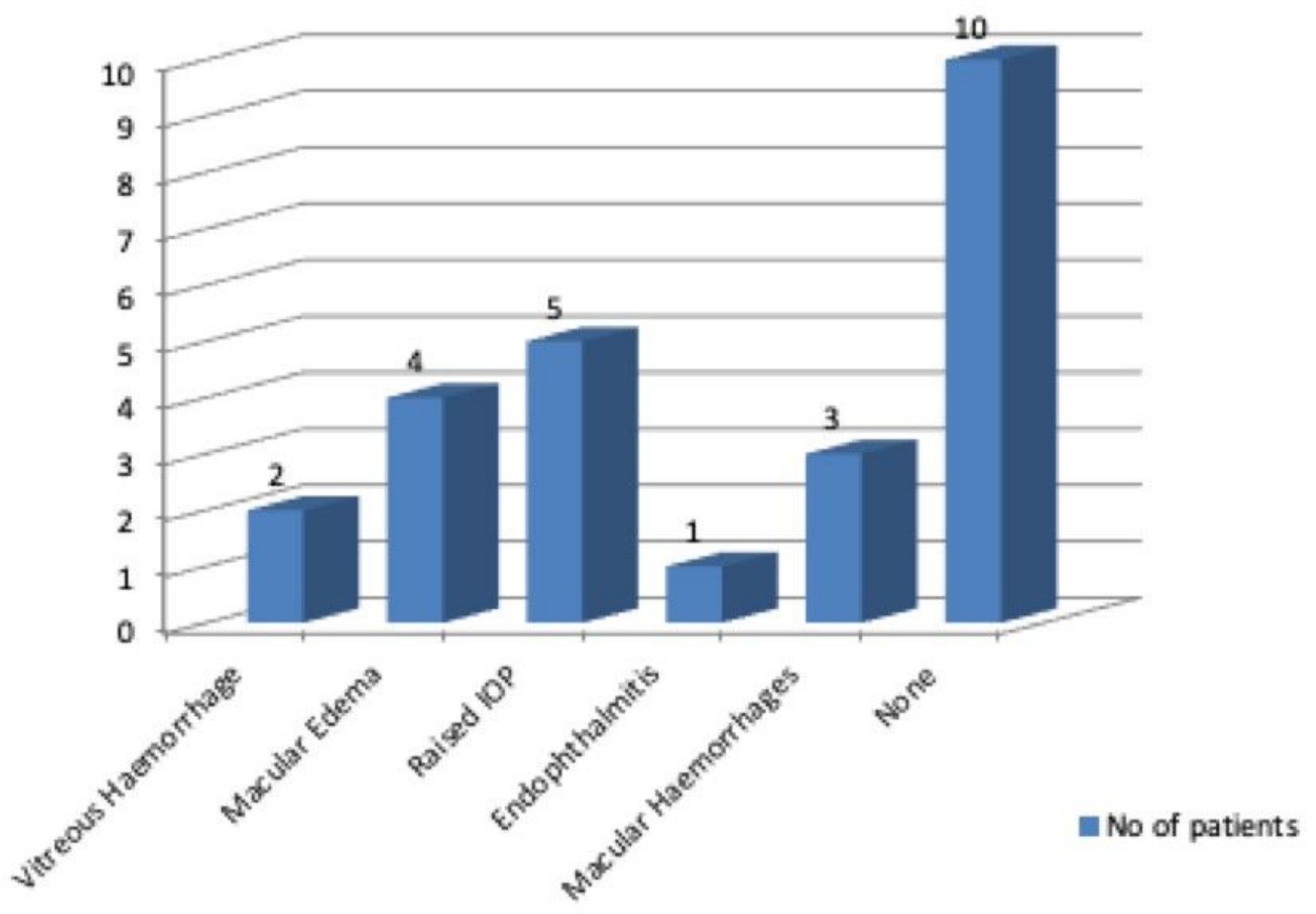

Figure 7

surgical complications 